\title{
DIVIMP modelling of tungsten impurity transport in ITER
}

\author{
K. Schmid, K. Krieger, A. Kukushkin, A. Loarte \\ Max- Planck-Institut für Plasmaphysik, EURATOM Association
}

\section{Abstract}

This paper presents a modeling study of the $\mathrm{W}$ erosion and plasma accumulation in ITER for different levels of first wall W coverage and plasma configurations using the Monte Carlo impurity transport code DIVIMP. Based on the ion fluxes from the background plasma solutions from B2/Eirene the $\mathrm{W}$ erosion is calculated by taking into account both $\mathrm{D}$ and impurity ions ( $\mathrm{C}, \mathrm{Ar}$ and $\mathrm{He}$ ). Based on these $\mathrm{W}$ erosion fluxes the W plasma concentrations are calculated. The calculations are performed for standard steady state divertor plasma geometry and for limiter geometry mimicking the ITER ramp up. The calculations show that in steady state divertor operation the $\mathrm{W}$ erosion and plasma concentrations are low even when the $\mathrm{W}$ first wall coverage is increased to a full W ITER. In limiter geometry due to the high electron temperatures close to the $\mathrm{W}$ limiter, strong $\mathrm{W}$ erosion is observed even for pure D plasmas. When seeded impurities are taken into account, intolerable $\mathrm{W}$ plasma concentrations of several percent are observed in the limiter plasma configuration.

PACS: 52.25.V, 52.65, 52.40.H

JNM-keywords: D0500, P0500

PSI-17 keywords: Edge plasma, Erosion \& Deposition, High-Z material, ITER, Tungsten

Author address: IPP Garching, Bereich MF, Boltzmannstrasse 2, 85738 Garching, Germany

Author e-mail: Klaus.Schmid@ipp.mpg.de

Presenting author: Klaus Schmid

Presenting author e-mail: Klaus.Schmid@ipp.mpg.de 


\section{Introduction}

In most of the current fusion experiments the plasma facing wall is made from low $Z$ materials $(\mathrm{C}, \mathrm{Be})$ despite their comparatively high erosion yield. The primary reason is that even if these impurities accumulate in the plasma core to a level of several percent they do not lead to significant radiation cooling of the plasma. In future devices like ITER or DEMO which are designed to operate at pulse lengths of up an hour or might even operate in continuous mode, the use of low $Z$ wall materials is not feasible. Apart from the issue of low- $Z$ element component lifetime problems due to excess erosion, co-deposition with $\mathrm{T}$ and the resulting tritium inventory are the primary reasons for switching to a high-Z low erosion material. The obvious choice for a high-Z first wall material is tungsten (W) since it has the highest surface binding energy $(8.68 \mathrm{eV})$ of all elements and thus the lowest erosion rate by light elements (D, $\mathrm{T}, \mathrm{He}$ ). The major problem introduced by the use of $\mathrm{W}$ is its high radiative cooling rate compared to $\mathrm{C}$, in particular at electron temperatures in the $\mathrm{keV}$ range as found in the plasma core. Thus the maximum allowable core $\mathrm{W}$ concentration $\left(\sim 10^{-5}\right)$ is roughly three orders of magnitude below that of $\mathrm{C}$ and therefore $\mathrm{W}$ erosion has to be kept at a minimum during operation and effective means are required to remove it from the plasma core. Keeping the core concentration low also requires a low impurity content in the plasma boundary. To still maintain a radiating plasma edge requires the seeding of medium- $\mathrm{Z}$ impurities ( $\mathrm{Ar}, \mathrm{Ne}$ ) into the plasma which have high cooling rates at the low electron temperatures in the edge plasma. The introduction of these seeded impurities could result in an increase of the $W$ erosion due to their higher mass and potentially higher impact energy compared to D. However, this increase in erosion could be compensated by a reduction in plasma temperature due to radiation cooling by the seeded impurities. Currently the use of W in ITER is restricted to the divertor baffles. However, if no viable removal methods for tritium co- 
deposited at the divertor are developed, a full $\mathrm{W}$ divertor target might become necessary. Furthermore, extending the use of $\mathrm{W}$ as plasma facing component (PFC) beyond the divertor in ITER to the main limiters or some parts of the first wall requires the evaluation of the associated risks for plasma contamination by means of plasma modelling. Because of this, the understanding of $\mathrm{W}$ erosion and its transport in the plasma edge in ITER are essential to predict the performance of ITER [1, 2].

Using the Monte Carlo impurity transport code DIVIMP [3] and plasma edge background solutions from B2-Eirene calculations for ITER [4], the erosion and transport of W in ITER was simulated. In divertor plasma geometry the influence of different levels of $\mathrm{W}$ first wall coverage on the $\mathrm{W}$ impurity plasma content was investigated. To estimate the erosion of a $\mathrm{W}$ limiter during ramp up calculations with an outboard limiter were performed.

\section{Calculation details}

The calculations presented here can be divided into two types with different plasma geometry: A divertor plasma with different levels of $\mathrm{W}$ coverage on the ITER first wall and a limiter plasma on an outboard toroidal $W$ limiter mimicking conditions during ITER ramp up. The equilibrium calculation grids and wall geometries used for both cases are shown in Fig. 1.

The background plasma for the divertor geometry was taken from B2/Eirene calculations $[5,6]$. The limiter case discussed here was intended to study the erosion and prompt re-deposition processes and the influence of $W$ self sputtering on the total $\mathrm{W}$ erosion yield during the ramp up phase of ITER. Since no B2/Eirene plasma background is available for the limiter case, a simple scrape off layer (SOL) model was used to generate $\mathrm{Te}, \mathrm{Ti}$ and $\mathrm{N}_{\mathrm{e}}$ for the computational grid. 
The B2/Eirene background plasmas available for the divertor case are summarized in Tab. 1. They differ in density, power into the SOL and the type of main radiating impurity. For $\mathrm{C}$ strike points the main impurity is $\mathrm{C}$ and for a full $\mathrm{W}$ divertor the main impurity is seeded Ar.

For the limiter case, a simple thermalized $\left(T_{e}=T_{i}\right)$ background plasma with no intrinsic impurities was generated in DIVIMP. The plasma parameters feature an exponential decay radially outward from the separatrix with a decay length $\lambda$ of $0.04 \mathrm{~m}$ calculated according to Eq.1.

$$
\lambda=\sqrt{D_{P E R P} * L_{C} / c_{s}}
$$

with $\mathrm{L}_{\mathrm{c}}=$ Connection length $(\sim 200 \mathrm{~m}), \mathrm{C}_{\mathrm{S}}=$ Sound speed $\left(\sim 10^{5} \mathrm{~m} \mathrm{~s}^{-1}\right)$ and $\mathrm{D}_{\text {PERP }}=$ perpendicular diffusion transport coefficient $\left(1 \mathrm{~m}^{2} \mathrm{~s}^{-1}\right)$.

From the separatrix to the core, the plasma parameters are assumed to increase linearly with radial distance. Along the field lines the plasma parameters were varied applying a solution for a conduction limited SOL according to [7] p.430.

At the separatrix the following plasma parameters were used: $T_{e}=150 \mathrm{eV}, \mathrm{n}_{\mathrm{e}}=$ $1 \times 10^{19} \mathrm{~m}^{-3}$, resulting in a maximum $T_{e}$ of $52 \mathrm{eV}$ and a maximum $\mathrm{D}^{+}$flux of $1.7 \times 10^{22} \mathrm{~m}^{-2} \mathrm{~s}^{-1}$ at the location where the separatrix touches the limiter surface. These parameters were chosen according to suggestions by the ITER modeling team (A. Kukushkin) who suggested that $T_{e}$ and $n_{e}$ at the separatrix during the limiter phase are expected to be not very different from the values during the divertor phase. Therefore the separatrix parameters from a low density, low power reference case (iter911) were used. Also the linear increase of $T_{e}$ and $n_{e}$ inside the separatrix were adjusted to match the plasma in the iter911 case.

As DIVIMP can only handle toroidally symmetric $2 \mathrm{D}$ wall geometries, the simulation describes the erosion of a W belt limiter in ITER. In reality the limiter will be not a 
toroidal but a poloidal limiter. Assuming that the connection length is the same order of magnitude for the poloidal limiter, the total plasma flux per meter toroidal and second $\left(\mathrm{m}\right.$-tor $\mathrm{s}^{-1}$ ) remains the same and thus also the total $\mathrm{W}$ erosion flux $\mathrm{m}$-tor $\mathrm{s}^{-1}$ is the same. If these assumptions hold, also self sputtering should be the same for the poloidal limiter.

DIVIMP was initially designed to simulate the erosion and transport of $\mathrm{C}$ in divertor and SOL regions of tokamaks. Therefore, it can only treat erosion at the divertor targets and is limited to one incident eroding and one target/impurity species. For the divertor cases $\mathrm{W}$ erosion occurs at the baffles and the main wall, which are not part of DIVIMP's calculation grid and thus the erosion of W from these surfaces cannot be handled directly by DIVIMP. Also the erosion by multiple plasma species (D-CX, D+, $\mathrm{He}^{+\mathrm{X}}, \mathrm{C}^{+\mathrm{X}}, \mathrm{Ar}^{+\mathrm{X}}$ ) cannot be handled by DIVIMP. Therefore the $\mathrm{W}$ erosion calculation had to be performed in a separate step. In the divertor geometry the particle fluxes of $\mathrm{D}-\mathrm{CX}, \mathrm{D}+, \mathrm{He}^{+\mathrm{X}}, \mathrm{C}^{+\mathrm{X}}, \mathrm{Ar}^{+\mathrm{X}}$ across the grid boundary, and the plasma parameters $\mathrm{Te}$, $\mathrm{Ti}$ at the grid boundary are known from the B2/Eirene calculations and can be extrapolated towards the wall. While the CX flux can be readily extrapolated towards the wall without having to account for attenuation processes, the extrapolation of the ion fluxes and plasma parameters $T_{e}, T_{i}$ to the wall is not straight forward. Since no validated physical model exists on how to extrapolate towards the wall, we assumed no attenuation at all during these calculations. This extrapolation is only necessary for parts of the baffle and for the entire main chamber where the calculation grid does not extend all the way to the actual wall. Therefore, the maximum erosion fluxes that were found at baffle locations close to the strike points are not affected by the ambiguity of this extrapolation. For the rest of the wall this extrapolation results in a overestimation of the actual wall ion fluxes and thus the resulting $\mathrm{W}$ plasma concentrations and erosion rates can be seen as an upper limit. In limiter geometry, 
the $D$ and Ar fluxes onto the limiter were calculated in a separate DIVIMP run where Ar was seeded from the top of the machine as indicated in Fig. 1. For the limiter cases no charge exchange information is available. However since the results in divertor configuration indicate that $\mathrm{W}$ erosion by $\mathrm{CX}$ is small compared to the erosion by impurity ions, the lack of CX information should not influence the results in limiter geometry.

Based on the CX and ion fluxes on the wall elements, the W-erosion flux was calculated using sputter yields from the revised Bohdansky formula [8]. The energy of an ion with charge state $q$ was calculated using $E_{I O N}=3 * q * T_{e}+2 * T_{i}$ [7] p. 79 and p. 69 and the CX energy was taken directly from the B2/Eirene background plasma solution. Based on this calculated distribution of the $\mathrm{W}$ erosion flux $\left(\mathrm{m}^{-2} \mathrm{~s}^{-1}\right)$ along the first wall, impurity launch probabilities along the first wall were calculated as input into DIVIMP. In DIVIMP W atoms are then launched with a spatial distribution according to these input probabilities. The particles are then followed through the subsequent impurity transport processes.

\section{W-erosion and plasma densities}

For the standard ITER configuration (W only at the baffles, $\mathrm{C}$ strike points) the erosion fluxes are very similar both in magnitude (within a factor of two) and poloidal distribution for the 6 different background plasmas. The highest erosion flux occurred at the transition from the vertical target plates to the curved baffles (see Fig. 2) with peak erosion fluxes of $1.5 \times 10^{19} \mathrm{~m}^{-2} \mathrm{~s}^{-1}$ ( $~ 8 \mathrm{~mm} /$ fyear).

The average $\mathrm{W}$ plasma densities calculated by DIVIMP based on these input erosion fluxes are in the order of $10^{14} \mathrm{~m}^{-3}$. The highest densities are found in the outer divertor where also the highest erosion rates were found. The plasma concentrations corresponding to these densities are of the order of $10^{-6}$ for the core plasma (pedestal to separatrix) and for the SOL (separatrix to outer boundary of calculation 
grid). In the divertor, the concentration is of the order of $10^{-4}$. For the high density cases the tungsten density in the divertor area is directly correlated to the power input. For the low density cases, however, there is no direct correlation. This is likely caused by differences in the B2/Eirene background plasma properties (temperature gradients, flow velocities etc.).

Most of the erosion occurs through highly charged $C$ ions and D contributes only through its charge exchange species due to the comparatively low plasma temperatures at the baffle region of 15 to $20 \mathrm{eV}$. Self sputtering of $\mathrm{W}$ is calculated at the divertor targets by DIVIMP but due to the low plasma temperatures of $\sim 5 \mathrm{eV}$ at the targets the influence of self sputtering is negligible for the divertor case.

When the $\mathrm{W}$ coverage of the first wall is increased to a full $\mathrm{W}$ divertor $\mathrm{C}$ no longer is the primary plasma impurity. Therefore for our investigation of the evolution of $\mathrm{W}$ impurity density as function of the $\mathrm{W}$ first wall coverage a B2/Eirene background solution with $\sim 1 \% \operatorname{Ar}$ as a seeded impurity was used (iter491). The erosion results for this Ar seeded case, iter491, with a full W divertor are very similar, both in poloidal distribution and in magnitude, to the $\mathrm{C}$ strike point cases. The highest erosion fluxes are again found at the transition from the baffle to divertor target (see Fig. 2).

Most of the erosion occurs through highly charged Ar ions and D only contributes through its charge exchange species due to the low plasma temperatures at the divertor and baffle region.

The W plasma densities and concentrations calculated by DIVIMP for the iter491 case based on these input erosion fluxes are identical within a factor of 2 to the values obtained for the $\mathrm{C}$ strike point divertor. This is due to the fast decay of the plasma temperature towards the strike point and the resulting decrease of the incident particle energy below the sputtering threshold as can also be seen from Fig. 
2. Thus, the increased $W$ area at the strike points does not act as a significant additional W source.

To investigate the influence of $\mathrm{W}$ usage outside the divertor, the $\mathrm{W}$ wall coverage was stepwise increased: full W divertor, full W divertor + inner wall, full W divertor + outer wall, full W ITER. The erosion flux $\left(\mathrm{m}^{-2}\right)$ increases as the first wall $\mathrm{W}$ coverage is increased for the iter491 Ar-case background plasma case as shown in Fig. 2. The highest erosion fluxes still occur within the divertor. The additional erosion of the main chamber wall only results in a factor 2 increase of the total $\mathrm{W}$ influx despite its larger surface area. The $\mathrm{W}$ plasma density resulting from these erosion fluxes is shown in Fig. 3 for different levels of wall coverage. For a full $\mathrm{W}$ divertor the plasma concentration increases by a factor of 2 and for a full W ITER first wall the plasma concentration is increased by a factor 5 .

These results are also supported by the experience in ASDEX Upgrade operating with W-coated PFCs. For divertor operation, experiments both in the initial W divertor experiment [9] and those done with a tungsten upper divertor in upper single null plasma configuration [10] showed similar $W$ erosion as a result of low-Z impurity impact.

For the limiter grid two sets of simulations were performed: One with a pure D plasma $\left(Z_{E f f}=1\right)$ and one with seeded Ar impurities. For the pure $D$ plasma, the erosion by $D$ ions was calculated in DIVIMP. The maximum erosion fluxes occur where the separatrix touches the $\mathrm{W}$ limiter surface where the highest electron temperatures $(\sim 50 \mathrm{eV})$ and particle fluxes $\left(10^{22} \mathrm{~m}^{-2} \mathrm{~s}^{-1}\right)$ occur. While the maximum erosion by $D$ alone of $1 \times 10^{17} \mathrm{~m}^{-2} \mathrm{~s}^{-1}$ is low compared to the divertor case, the influence of $\mathrm{W}$ self sputtering increases the $\mathrm{W}$ erosion flux by two orders of magnitude to $1 \times 10^{19} \mathrm{~m}^{-2} \mathrm{~s}^{-1}$. This strong influence of self sputtering is due to the high electron temperature, which results in highly charged $\mathrm{W}$ ions with keV impact energies. The resulting $\mathrm{W}$ plasma 
concentrations with the contribution of self sputtering range from $10^{-3}$ close to the limiter to $10^{-5}$ in the core and main plasma. The high core concentration is not only due to the high erosion yield but also due to the close vicinity of the limiter surface to the separatrix allowing for easy penetration of $W$ into the core. A comparison to a calculation without self sputtering shows that self sputtering increases the density of W in the plasma by an order of magnitude throughout the calculation grid.

To test the influence of Ar sputtering, two additional sets of DIVIMP calculations were performed: In the first calculation Ar was seeded from the top of the machine into the

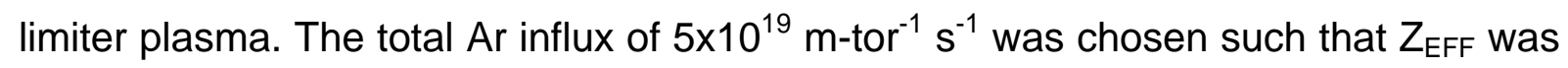
increased from 1.0 for the pure D plasma case to 1.3 including Ar. This resulted in maximum $\mathrm{Ar}$ concentrations in the $0.1 \%$ range in the plasma, which is also what is expected for ITER derived from the required radiation cooling [11]. From this calculation the spatially resolved $\mathrm{Ar}$ ion flux distribution along the $\mathrm{W}$ limiter was obtained for each charge state. Together with the plasma temperature at the limiter surface, the W-erosion flux due to each Ar ion species was calculated. By adding the contribution of erosion by $\mathrm{D}^{+}$the total W-erosion flux distribution along the limiter surface due to $\mathrm{Ar}$ and $\mathrm{D}$ bombardment was obtained and is summarized in Fig. 4 for the different sputtering conditions. From Fig. 4 it follows that the seeding of Ar results in an increase of the total $W$ erosion by a factor of 4 without taking self sputtering into account. Using this $\mathrm{W}$ erosion flux distribution, the $\mathrm{W}$ plasma impurity concentration was calculated in a second DIVIMP run. The W plasma concentrations even without the influence of self sputtering reach \% levels. Including $\mathrm{W}$ self sputtering results in a plasma with $\mathrm{W}$ as the majority species and a $Z_{E F F}$ of 20 . Of course this is not a realistic result since the trace particle approximation used by DIVIMP is clearly violated at these $\mathrm{W}$ concentrations. However, these calculations still indicate that the introduction of low $\mathrm{Z}$ impurities into the hot limiter plasma will lead to excessive $\mathrm{W}$ 
sputtering and thus to unacceptably high $\mathrm{W}$ plasma concentrations during ramp up. The question remains if the seeded Ar can sufficiently cool the plasma to avoid excessive W sputtering. The cooling efficiency of seeded Ar can not be simulated in DIVIMP and would require self consistent simulations including radiation cooling using an integrated code like B2/Eirene.

\section{Conclusions}

The erosion of $\mathrm{W}$ from the ITER first wall and subsequent transport in the SOL was simulated for different ITER reference background plasma scenarios using the Monte-Carlo impurity transport code DIVIMP. In these calculations the influence of different $\mathrm{W}$ first wall coverage levels and $\mathrm{C}$ or Ar impurity ions on W erosion and plasma accumulation was investigated. Two different plasma geometries were considered: Detached divertor and limiter configurations. The divertor plasmas represent normal ITER flat top operation while the limiter plasmas approximate the ITER ramp up. For the standard ITER configuration with $W$ only at the baffles, the plasma concentration in the core is well below the critical value of $10^{-4}$. Increasing the W first wall coverage to a full W divertor increases plasma concentrations by a factor of 2 and for a full W ITER first wall the plasma concentration is increased by a factor $5 . \mathrm{W}$ is primarily eroded by highly charged low $\mathrm{Z}$ impurities ( $\operatorname{Ar}$ or $\mathrm{C}$ ).

The limiter plasma configuration is much more critical: Even under the ideal conditions of a pure $\mathrm{D}$ plasma $\left(\mathrm{Z}_{\mathrm{EFF}}=1\right)$ the erosion of $\mathrm{W}$ close to the separatrix was almost at the run away self sputtering threshold for $T_{e}=150 \mathrm{eV}$ and $n_{e}=1 \times 10^{19} \mathrm{~m}^{-3}$ at the separatrix. Moreover the eroded $\mathrm{W}$ has a high probability of penetrating into the confined plasma due to the close vicinity of the location of maximum erosion to the separatrix. Adding seeded Ar impurities increases the W erosion and plasma concentrations by orders of magnitude resulting in percent level $\mathrm{W}$ concentrations. This shows that seeded impurities like $\operatorname{Ar}$ may critically deteriorate plasma 
performance and limiter lifetime particularly during the ramp up phase. To avoid this, the seeded impurities must cool the plasma sufficiently to compensate the enhanced sputtering. While experience in AUG has shown that this compensation is possible for low $T_{e}$ divertor operation [10], it seems more problematic from Fig. 4 to achieve high enough cooling for the high $\mathrm{T}_{\mathrm{e}}$ limiter operation.

From the calculations in divertor plasma configuration one can conclude that operating a full $\mathrm{W}$ divertor or even ITER with a full $\mathrm{W}$ first wall in detached divertor mode will not be limited by $W$ erosion. The limiter plasma calculations show, however, that performing a plasma ramp up on a $\mathrm{W}$ limiter could result in excessive W sputtering, and accumulation in the plasma core.

\section{Acknowledgement}

This work was partly funded by the task TW5-TPP-TUNMOD of the EFDA technology program. 


\section{Figure and table captions}

Tab. 1 Summary of plasma B2/Eirene plasma backgrounds for the divertor plasma geometry

Fig. 1 The wall and calculation grid for the divertor a.) and limiter b.) plasma geometry.

Fig. 2 Calculated poloidal $W$ erosion flux distribution for different $W$ first wall coverage levels.

Fig. 3 Increase of the average W plasma density in the Divertor, main SOL and Core as function of the $W$ first wall coverage.

Fig. 4 W erosion flux due sputtering by $D$ and seeded Ar of a $W$ limiter 
Tables

\begin{tabular}{|c|c|}
\hline Case ID & Power into SOL [MW] \\
\hline \multicolumn{2}{|l|}{ High density Cases: } \\
\hline iter881 (C strike points) & 130 \\
\hline iter812 (C strike points) & 100 \\
\hline iter877 (C strike points) & 86 \\
\hline iter491 (Full W divertor, Ar seeding) & 130 \\
\hline \multicolumn{2}{|l|}{ Low density Cases: } \\
\hline iter864 (C strike points) & 130 \\
\hline iter884 (C strike points) & 100 \\
\hline iter911 (C strike points) & 86 \\
\hline
\end{tabular}

Tab. 1 


\section{Figures}

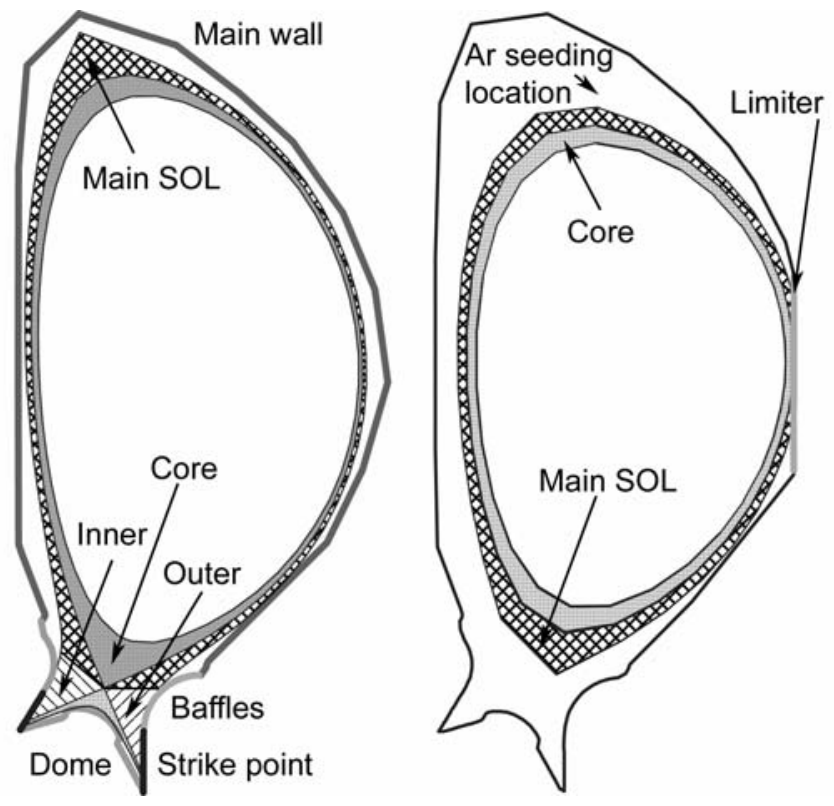

Fig. 1

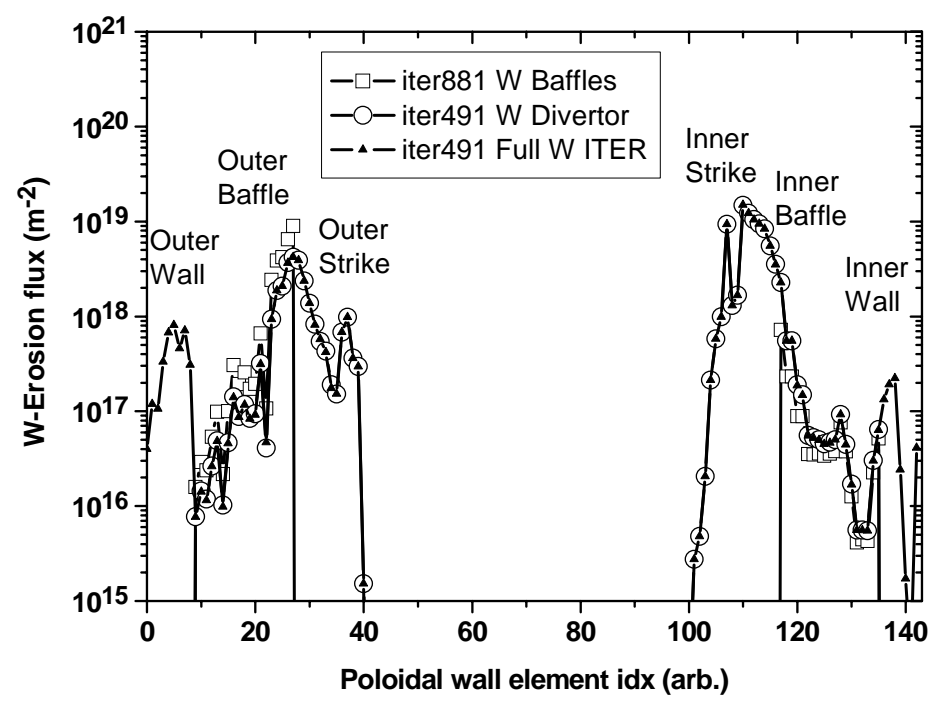

Fig. 2 


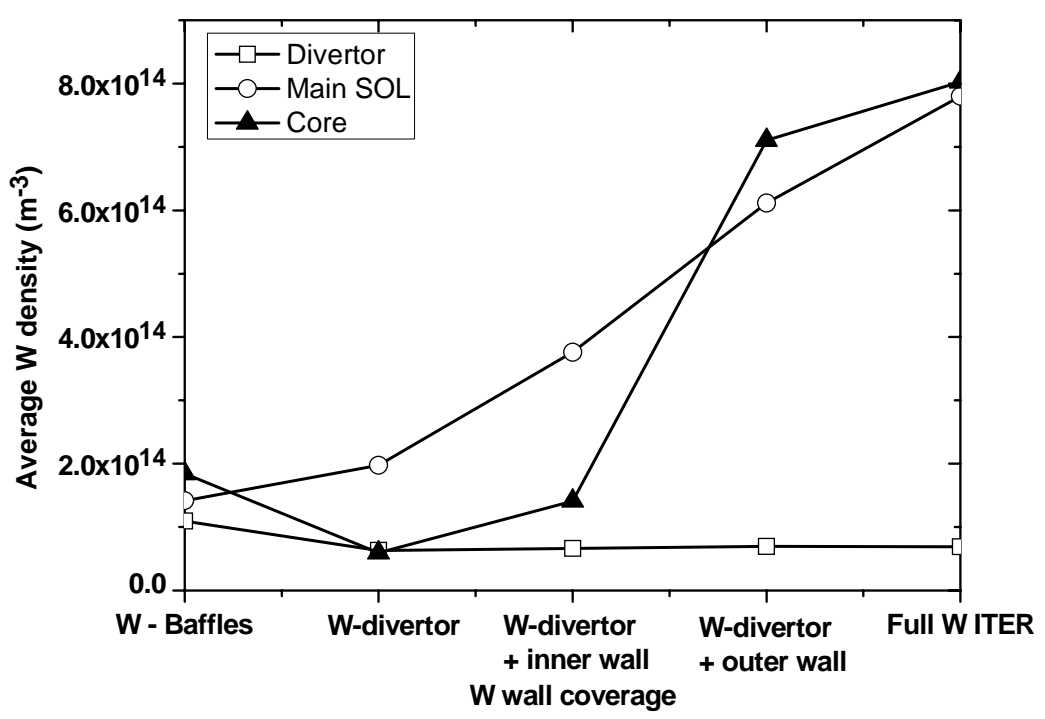

Fig. 3

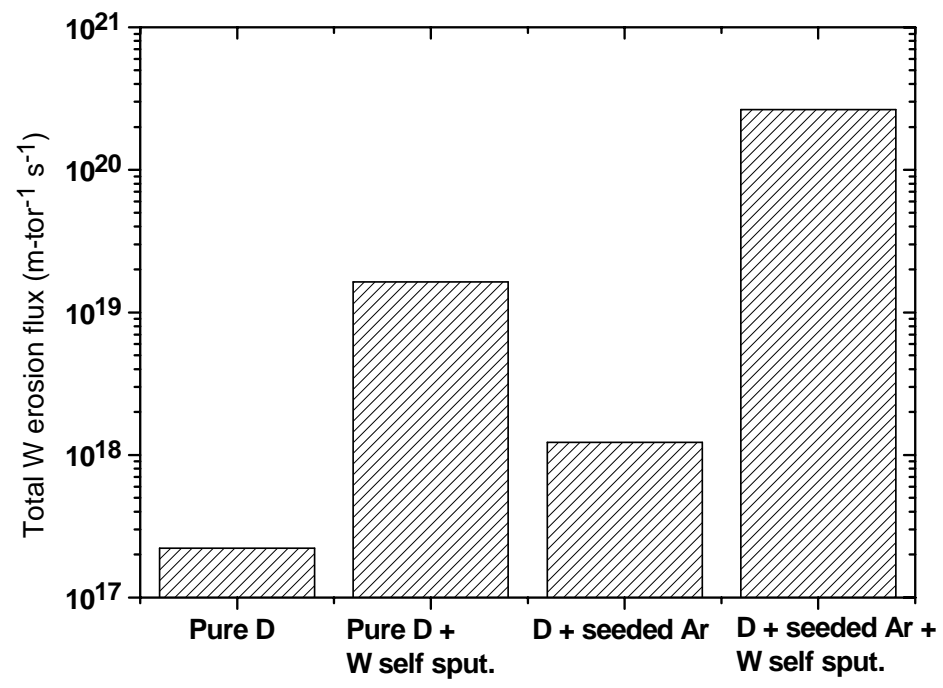

Fig. 4 


\section{Literature}

1 Geier, A., et al., A., J. Nuc. Mat. 313-316 (2003), 1216.

2 Federici, G., et al., A., J. Nuc. Mat. 313-316 (2003), 11

3 P.C. Stangeby and J. D. Elder, J. Nucl. Mater. 196-198 (1992), 258

4 A.S. Kukushkin, H.D. Pacher, G. Janeschitz, A. Loarte, D.P. Coster, et al., Nucl. Fus., 42 (2002), 187.

5 A.S.Kukushkin, H.D.Pacher, D.P.Coster, et al, J. Nucl. Mater. 337-339 (2005), 50

6 A.S.Kukushkin, H.D.Pacher, Plasma Phys. Control. Fusion 44(2002), 931

7 P. C. Stangeby, „The plasma boundary of magnetic fusion devices“, IOP Pulishing (2000)

8 J. Bohdansky, Nucl. Inst. Meth. B 2, (1984), 587

9 R. Neu, K. Asmussen, K. Krieger, A. Thoma, H-S. Bosch, S. Deschka, R. Dux, et. Al, Plasma Phys. Control. Fusion 38 (1996), A165-A179

10 R. Neu, R. Dux, A. Kallenbach, T. Pütterich et.Al., Nucl. Fusion 45 (2005, 209

11 G. Becker, Nuclear Fusion, Vol. 39, No. 7, (1999), 937 